\title{
The Heavy Photon Search experiment at Jefferson Laboratory
}

\author{
Marzio De Napoli ${ }^{1, a}$ for the HPS Collaboration \\ ${ }^{1}$ INFN - Sezione di Catania, Via Santa Sofia 64, 95123 Catania, Italy
}

\begin{abstract}
Many beyond Standard Model theories predict a new massive gauge boson, aka "dark" or "heavy photon", directly coupling to hidden sector particles with dark charge. The heavy photon is expected to mix with the Standard Model photon through kinetic mixing and therefore couple weakly to normal charge. The Heavy Photon Search (HPS) experiment will search for the heavy photon at the Thomas Jefferson National Accelerator Facility (JLab), in the mass range $20-1000 \mathrm{MeV} / \mathrm{c}^{2}$ and coupling to electric charge $\epsilon^{2}=\alpha^{\prime} / \alpha$ in the range $10^{-5}$ to $10^{-10}$. HPS will look for the $e^{+} e^{-}$decay channel of heavy photons radiated by electron Bremsstrahlung, employing both invariant mass search and detached vertexing techniques. The experiment employs a compact forward spectrometer comprising silicon microstrip detectors for vertexing and tracking and an electromagnetic calorimeter for particle identification and triggering.
\end{abstract}

\section{Introduction}

The existence of an additional U(1) gauge symmetry in nature is predicted by several beyond the Standard Model theories $[1,2]$. The associated gauge boson $A^{\prime}$, called "dark" or "heavy photon", can mix kinematically with the photon through quantum loops of heavy particles that carry both the standard model hypercharge and the dark force equivalent $[3,4]$. This kinetic mixing results in an effective interaction $\epsilon e A_{\mu}^{\prime} J_{E M}^{\mu}$ of the $A^{\prime}$ to the electromagnetic current $J_{E M}^{\mu}$, suppressed relative to the electron charge $e$ by a factor $\epsilon$. Since heavy photons couple to electrons, they can be produced by electron Bremsstrahlung off heavy nuclear targets and can decay to $e^{+} e^{-}$pairs.

Heavy photons have been suggested as a possible explanation of several puzzling experimental observations [6, 7]. The decay or annihilation of dark matter charged under this dark force can explain the high-energy positron excess in the cosmic-ray flux measured by PAMELA [8], FERMI [9] and AMS-02 [10]. The lack of excess in the antiproton-to-proton ratio suggests a mass of $A^{\prime}$ lower than $\simeq 2 \mathrm{GeV}$ [11]. In this scenario, $A^{\prime}$ represents a possible "portal" between the Standard Model and the dark matter sector. Besides having a possible connection to dark matter, an $A^{\prime}$ could explain the discrepancy between the measured and calculated value of the anomalous magnetic moment $(g-2)_{\mu}$ of the muon [12].

Current phenomenology highlights an $A^{\prime}$ in a particular mass range with $\mathrm{MeV}$ - to $\mathrm{GeV}$-scale mass and suggests that the coupling to electric charge, $e \epsilon$, has $\epsilon^{2}$ in the range of $10^{-10}-10^{-6}$. Although a significant part of this masscoupling parameter space has already been excluded by several experiments (see Ref. [13] for a complete review), a large region remains unexplored. A new generation of

a e-mail: marzio.denapoli@ct.infn.it dedicated high-luminosity fixed-target experiments offers a unique opportunity to probe this unknown region [14]. Among them, the Heavy Photon Search (HPS) experiment will search for the existence of a heavy photon in the mass range $20-1000 \mathrm{MeV} / \mathrm{c}^{2}$ and coupling $\epsilon^{2}$ between $10^{-10}$ and $10^{-5}$.

\section{The HPS Experiment}

HPS is a fixed-target experiment that will run in experimental Hall B of the Thomas Jefferson National Accelerator Facility (JLab), using the CEBAF high-intensity electron beam. The beam, impinging on a thin $\left(0.25 \% X_{0}\right)$ Tungsten target foil, can produce heavy photons through a process analogous to Bremsstrahlung, and the $A^{\prime}$ can then decay to $e^{+} e^{-}$pairs (Fig. 1). Since the coupling of heavy photons to $e^{+} e^{-}$pairs is much weaker than the canonical electromagnetic coupling, heavy photon production is buried in a huge background of pairs from virtual photons. By reconstructing the momenta of the $e^{+} e^{-}$pairs, HPS will search for a narrow resonance in the invariant mass spectrum over the quantum electrodynamics (QED) background (the traditional "bump-hunting"). The main QED background reactions are the radiative trident production and the Bethe-Heitler process (Fig. 1). Although the Bethe-Heitler process has much larger total cross-section than the radiative one, it can be significantly reduced by exploiting its different kinematics with respect to the $A^{\prime}$ one. Instead, the radiative trident process is an irreducible background, with the same kinematics as the signal. The latter is characterized by a very-forward emitted $A^{\prime}$ that carries most of the beam energy $E$. Therefore, the $A^{\prime}$ decay products are boosted forward with typical polar angles $\sim M_{A^{\prime}} / E$, of the order of few degrees. Moreover, depending on $M_{A^{\prime}}$ and $\epsilon, A^{\prime}$ can be long-lived, with a 
a)

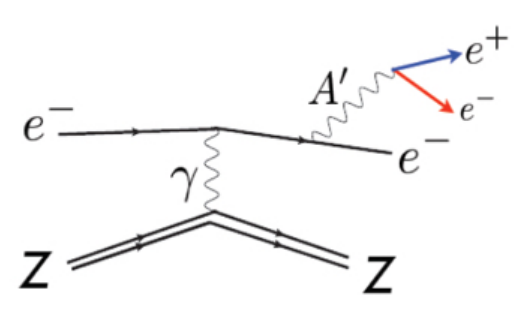

b)

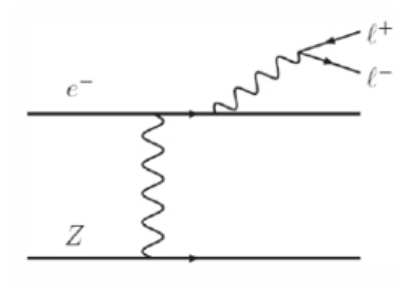

"Radiative"

c)

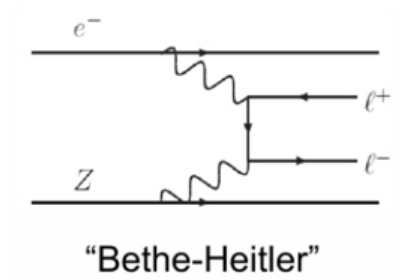

Figure 1. (a) Feynman diagram for radiative $A^{\prime}$ electroproduction and $A^{\prime}$ decay to $e^{+} e^{-}$pairs. Diagrams of radiative $(b)$ and Bethe-Heitler (c) QED reactions, the primary background for the $A^{\prime}$ search, are also shown.

distinct secondary decay vertex detached from the target. HPS will also use this signature to distinguish the signal from the $\mathrm{QED} e^{+} e^{-}$pairs promptly produced in the target.

\subsection{Experimental setup}

The HPS detector [15] was designed to match the experimental requirements dictated by the search strategies and the signal kinematics discussed above. Bump hunting requires excellent track reconstruction for electrons and positrons with a good angular and momentum resolution, which is most easily accomplished with charged particle spectrometry. Detached vertexing requires the tracking detectors to be placed just downstream of the target to minimize extrapolation errors and optimize vertex resolution. Detectors must be also placed as close to the beam direction as possible to cover with high acceptance the forward angular region. At the same time, however, the detectors must avoid a region of intense electron flux caused by the small angle multiple coulomb scattering in the target and the horizontal dispersion in the magnetic field of the analyzing magnet of the electrons which have radiated energy in the target. The resulting "dead zone" has an angular range of $\theta_{y}<15 \mathrm{mrad}$ ( $y$ is the vertical dimension). This splits the apparatus into upper and lower halves. In addition, the beam is transported in vacuum through the tracker to minimize beam-gas interaction backgrounds. The high duty-cycle, high-quality and high-intensity CEBAF electron beam is also crucial to generate large integrated luminosities while keeping beam-related background under control. Even so, the occupancies of sensors near the beam plane are high, dominated by the multiple Coulomb scattering of the beam, so high-rate detectors, a fast trigger, and excellent time tagging are required to minimize their impact.

The HPS detector setup is shown in Fig. 2. It is made of a $\sim 1 \mathrm{~m}$ long Silicon Vertex Tracker (SVT) inside an analyzing dipole magnet, to reconstruct charged particle trajectories and vertexes, and a fast lead-tungstate electromagnetic calorimeter (ECal) to measure particle energies, identify the $e^{+} e^{-}$pairs, and generate the trigger of the experiment.

The SVT is made of 6 layers of pairs of closely-spaced silicon microstrip sensors mounted back-to-back to form a module. A small stereo angle between the two layers (50 or $100 \mathrm{mrad}$ ) provides three-dimensional tracking and vertexing. The SVT must minimize the material budget in the tracking volume to reduce multiple scattering and optimize both the invariant mass and vertex resolutions that directly affect the experimental sensitivity. Moreover, the signal yield for long-lived $A^{\prime}$ is very small, so the rejection of prompt vertices must be exceedingly pure, of the order of $10^{-7}$, in order to eliminate all prompt background tails. To achieve the required vertexing performance the first layer of the SVT must be placed no more than about $10 \mathrm{~cm}$ downstream of the target. At that distance, it is found that the active region of a sensor can be placed as close as $1.5 \mathrm{~mm}$ from the center of the beam, to maximize low-mass $A^{\prime}$ acceptance with decay products nearly collinear with the beam axis. Sensors are readout by the APV25 chip, developed for the CMS experiment at the CERN LHC [16], with a custom DAQ system based on the Advanced Telecom Communications Architecture (ATCA) technology.

The ECal is made of $442 \mathrm{PbWO}_{4}$ crystals, $16 \mathrm{~cm}$ thick, with a $13.3 \times 13.3 \mathrm{~mm}^{2}\left(16 \times 16 \mathrm{~mm}^{2}\right)$ front (rear) face. In each half of the detector, crystals are arranged in five rows of 46 crystals, except the one closest to the beam that has only 37 crystals. The light from each crystal, approximately 120 photons/MeV, is read out by largearea Avalanche PhotoDiodes (APD) glued to the back face. Signals from the APDs are read and amplified using custom-made pre-amplifiers before being sent to the JLab Flash ADC boards for digitization and processing. The onboard FPGA processes the digitized samples to provide information about the energy and the timing associated with each hit. This information, available during data-taking, is exploited by an advanced triggering algorithm matched to the foreseen $A^{\prime}$ signal: one high-energy electromagnetic shower in each ECal half.

\subsection{HPS reach}

Figure 3 shows the HPS reach in the mass-coupling parameter space, together with the exclusion limits from other 


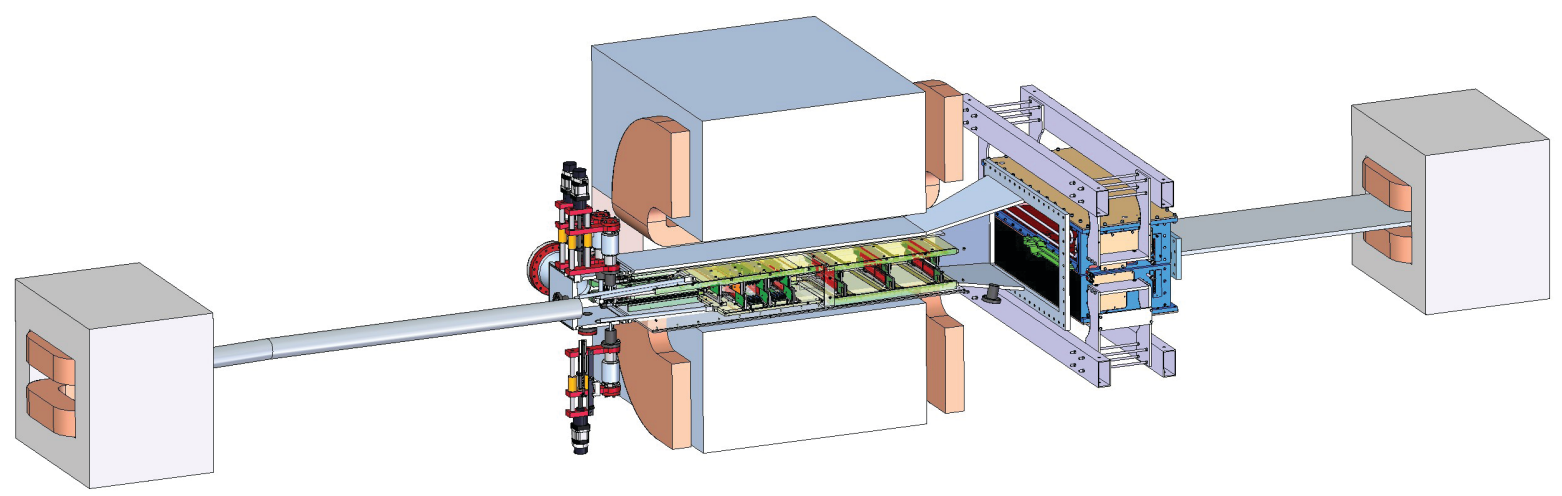

Figure 2. HPS detector setup. From left to right: the first dipole magnet, the target system, the SVT inside the analyzing magnet, the ECal and the third dipole magnet.

experiments and the constraint from the electromagnetic momentum $a_{e}$ (red band). The reach was computed by assuming a one-week run at $1.1 \mathrm{GeV}$, a one-week run at 2.2 $\mathrm{GeV}$, and a two-week run at $4.4 \mathrm{GeV}$. The two regions in the reach plot correspond to the two analysis techniques used in HPS: the resonance search at higher $\epsilon^{2}$ and the detached vertex search at lower $\epsilon^{2}$. The latter region is particularly difficult to reach and can be uniquely explored by HPS. Indeed, on one side, it corresponds to low $\epsilon^{2}$ and thus small cross sections, out of reach from collider experiments. On the other side, the relatively short decay length $(<1 \mathrm{~m})$ of $A^{\prime}$ in this region, makes it out of reach also from beam-dump experiments, where thick absorbers are used.

\section{References}

[1] Feldman D., Kors B. and Nath P., Phys. Rev. D 75, 023503 (2007)

[2] Andreas S., Goodsell M. and Ringwald A., Phys. Rev. D 87, 025007 (2013)

[3] Galison P. and Manohar A., Phys. Lett. B 136, 279 (1984)

[4] Holdom B., Phys. Lett. B 166, 196 (1984)

[5] Essig R., Schuster P. and Toro N., Phys. Rev. D 80, 015003 (2009)

[6] Arkani-Hamed N., Finkbeiner D.P., Statyer T.R., Weiner N., Phys. Rev. D 79, 015014 (2009)

[7] Pospelov M. and Ritz A., Phys. Lett. B 671, 391-7 (2009)

[8] Adriani O. et al., Nature 458, 607 (2009)

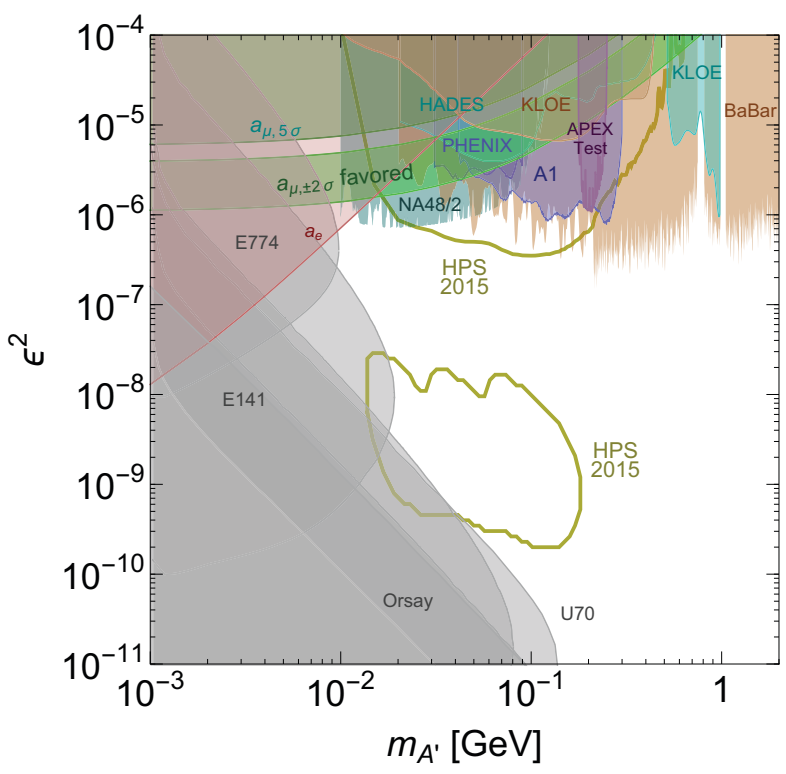

Figure 3. The HPS projected reach corresponding to the $2 \sigma$ limit (yellow line). The green band $a_{\mu} \pm 2 \sigma\left(a_{\mu} \pm 2 \sigma\right)$ corresponds to the region where the $A^{\prime}$ can explain the discrepancy between the experimental value of the muon anomalous magnetic moment, considering a $2 \sigma(5 \sigma)$ error, and the corresponding Standard Model calculation.

[9] Ackermann M. et al. (Fermi LAT Collaboration), Phys. Rev. Lett. 108, 011103 (2012)

[10] Aguilar M. et al. (AMS Collaboration), Phys. Rev. Lett. 110, 141102 (2013) 
[11] Adriani O. et al., Phys. Rev. Lett. 102, 051101 (2009)

[12] Pospelov M., Phys. Rev. D 80, 095002 (2009)

[13] Essig R. et al., "Dark Sectors and New, Light, Weakly-Coupled Particles" arXiv:1311.0029v1.

[14] Bjorken J.D., Essig R., Schuster P. and Toro N., Phys. Rev. D 80, 075018 (2009)
[15] Battaglieri M. et al. (HPS Collaboration), Nucl. Instr. and Meth. A 777, 91-101 (2015)

[16] French M., Jones L., Morrissey Q., Neviani A., Turchetta R. et al., Nucl. Instr. and Meth. A 466, 359-65 (2001) 